\title{
Evaluación de las emisiones de vapor mercurial en procesos de amalgamado artesanal: caso Cantón Ponce Enríquez, Provincia del Azuay
}

\author{
Galo Carrillo R., Ana Astudillo A. \\ Centro de Estudios Ambientales (CEA) y Dirección de Investigación de la Universidad de Cuenca \\ (DIUC), Cuenca, Ecuador
}

Autor para correspondencia: galo.carrillo@ucuenca.edu.ec

Fecha de recepción: 18 de noviembre de 2011 - Fecha de aceptación: 28 de diciembre de 2011

\begin{abstract}
RESUMEN
La minería a pequeña escala aplicada en varias localidades del Cantón Ponce Enríquez, Provincia del Azuay, Ecuador, emplea comúnmente el proceso de amalgamación con mercurio donde éste se adhiere a las partículas de oro contenidas en el material de mina, posteriormente la amalgama obtenida se vaporiza por calentamiento. Durante el proceso, los mineros y cualquier persona en la cercanía, se encuentran en peligro de inhalar vapores de mercurio. En este estudio se examinó la variación de la concentración de vapor de mercurio como una función de la distancia desde punto focal de quema, en cuatro localidades diferentes. Los resultados revelan que los niveles de vapor de mercurio emitidos durante la separación térmica de la amalgama, exceden ampliamente las normas internacionales y sus límites de salud y seguridad para distancias menores a un metro del punto de generación. En distancias superiores, se registra una notable disminución de la concentración de vapor mercurial. Las observaciones del estudio podrán ser instrumentos para la formulación de políticas y regulaciones que mitiguen los peligrosos efectos de la inhalación de mercurio.
\end{abstract}

Palabras clave: Minería artesanal del oro, amalgama de mercurio, vapor de mercurio.

\begin{abstract}
Small-scale mining as applied at several locations in the Canton Ponce Enriquez, located in the Province of Azuay, Ecuador, use commonly the mercury amalgamation process whereby elemental mercury is mixed with silt or ore dust that contain tiny pieces of gold. The amalgam is heated and his mercury evaporates, leaving pure gold or silver. During the process miners and anyone else in the vicinity are at risk of inhaling mercury. In this study the variation in the concentration of mercury vapor was examined as a function of the distance from the focal point of heating at four different locations. Results reveal that mercury vapor levels released during the amalgam thermal separation largely exceed the international norms and security and health limits within a distance of one meter of the focal point. At further distance a notable decrease in the concentration of mercury vapor is recorded. The observations will be instrumental for the formulation of policies and regulations that mitigate the hazardous effects of the inhaling of mercury.
\end{abstract}

Keywords: Artisanal gold mining, mercury amalgamation, mercury vapor.

\section{INTRODUCCIÓN}

\subsection{La importancia de la minería aurífera artesanal en Ecuador}

La pobreza y aprovechamiento de los recursos minerales disponibles han contribuido mundialmente al incremento de la minería a pequeña escala o conocida como "artesanal"; a pesar de que no se conoce con exactitud la dimensión de esta actividad, se reporta que entre el 20 a $30 \%$ de la producción 
mundial de oro (entre $500 \sim 800$ toneladas por año) se producen bajo este régimen artesanal en al menos 50 países en desarrollo (Swain y col., 2007). Además se conoce que entre 80 a 100 millones de personas son dependientes directos de los beneficios económicos de la minería a pequeña escala, y entre 13 a 15 millones de artesanos mineros son los que generan la producción anual de oro (Spiegel, 2009), esta actividad libera al medioambiente entre 800 a 1000 toneladas anuales de mercurio en forma directa de desperdicio del proceso y de los cuales 300 toneladas aproximadamente son emitidas en forma de vapor a la atmósfera (Swain y col., 2007). Con respecto de la situación minera en el Ecuador, se ha estimado que el aporte de la minería al PIB en los últimos años no supera el $0,35 \%$, y el mismo ha declinado desde esta cifra desde 2001, al 0,25\% en 2007 (Cisneros, 2008; MEM, 2007). Así mismo, en relación al empleo, la minería a gran escala generó en 2004 unas 4.871 plazas de trabajo, frente a las 84.280 que reporta la minería a pequeña escala en 2006 según datos de la Cámara de Pequeña Minería de Pichincha (MEM, 2007). Se comprende entonces que a pesar de generar un PIB relativamente bajo, estas actividades generan una significativa cantidad de empleo en las zonas del Ecuador donde se aplican. En lo referente específicamente a la minería extractiva del oro, según el Sistema de Administración de Derechos Mineros (SADMIN), se ha extraído más de $23.000 \mathrm{~kg}$ de oro desde el año 2000 al 2005 (MEM, 2007). Actualmente, el Gobierno Ecuatoriano plantea la creación de la Empresa Nacional Minera, como sociedad de derecho público autónoma, que agruparía posiblemente a los artesanos mineros y fomentaría el desarrollo sustentable de sus microempresas, la misma estaría regulada bajo el Ministerio de Recursos Naturales No Renovables del Ecuador, y por medio de la Agencia de Regulación y Control Minero, como entidad de control de las actividades actuales.

\subsection{Localización y perfil del personal activo en la minera artesanal en el Sur del Ecuador}

La actividad minera artesanal se encuentra en un lento desarrollo y ofrece sustento a hogares de bajos recursos económicos de las localidades donde se asientan sus concesiones. Desde el período precolombino, el Ecuador cuenta en su territorio con minas de minerales preciosos, ubicados principalmente al sur del país en cuatro regiones principales: Ponce Enríquez, Santa Rosa, PortoveloZaruma y Nambija. El Cantón Ponce Enríquez, está ubicado en la provincia del Azuay, tiene como principal fuente de recursos la actividad minera, diligencia a la que se han dedicado la mayoría de sus habitantes; según los datos del último censo del año 2001 se manifiesta que el 35\% de la población del cantón se dedica a la extracción de minerales metalíferos, seguido de un 32\% que se dedican a la agricultura y ganadería (INEC, 2001). Los yacimientos auríferos localizados en este cantón, se constituyen en los más importantes y productivos del Ecuador en la actualidad, y a pesar de algunas mejoras en los últimos años, la explotación del oro está dominada por operaciones artesanales y semimecanizadas, con poca supervisión gubernamental y asesoría técnica especializada.

En un estudio preliminar se establece que el $100 \%$ del personal activo en la minería artesanal de la zona investigada es de género masculino y la mayor parte trabaja como barretero o personal de perforación de la mina. Las edades en fluctúan entre 15 y 30 años de edad y el $70 \%$ alcanza un nivel de educación primaria. Menos del 25\% de los trabajadores emplean directamente productos químicos en el proceso, y en su mayoría lo que usan son precursores para explosión y dinamita en las actividades de voladura de roca en las minas. También menos del $10 \%$ han tenido contacto directo con mercurio para amalgamación. Las cantidades empleadas de este reactivo fluctúan entre los 600 a 1000 gr semanales, según la necesidad del proceso. Adicionalmente solo el $12 \%$ de la población investigada hace uso de la mascarilla para gases y partículas finas como equipo de protección personal, mientras que más del $90 \%$ de los encuestados desconoce de otras tecnologías de extracción de oro que sean menos riesgosas para la salud y el medioambiente. Se percibe falta de interés de parte de ellos para un cambio tecnológico que mejore las condiciones actuales de trabajo.

\subsection{Proceso artesanal}

Durante el proceso de obtención del oro, se dan una serie de operaciones, entre ellas la adición de mercurio metálico $(\mathrm{Hg})$, proceso que recibe el nombre de amalgamación, la misma que consiste en una mezcla mecanizada de mercurio líquido con el material de mina finamente molido, en el cual las partículas de mercurio se adhieren a las de oro. Según PNUMA (2005) se utilizan entre 1 a $2 \mathrm{~kg}$ de 
mercurio para extraer un kg de oro. Para la separación del material se emplea la centrifugación, luego de este proceso se da lugar a la concentración y recuperación de oro, para esto se calienta la amalgama hasta una temperatura de $700^{\circ} \mathrm{C}$ con soplete de gas GLP, en presencia o no, de ácido nítrico $\left(\mathrm{HNO}_{3}\right)$ el cual actúa como un agente solvente de impurezas (óxidos) y mejora la calidad del oro que será obtenido por quema posterior de la amalgama. El mercurio se evapora y es expulsado a la atmósfera circundante en la mayoría de los casos, y en ocasiones es recuperado hasta un $90 \%$ cuando se emplean campanas o retortas cerradas. En muchos casos todavía se quema mercurio al aire libre y, en otros se utilizan retortas para la destilación de amalgama; se tiene conocimiento de iniciativas para la promoción de centros de quemado, pero han tenido un éxito muy limitado (Sandoval, 2001). El oro obtenido muestra una alta concentración $(>99.5 \%)$ y puede ser vendido directamente a joyerías. El peso de las emisiones de mercurio no recuperado es muy alto; estas emisiones son inhaladas por los trabajadores mineros y se precipitan sobre la zona y materiales de trabajo, el terreno y la vegetación. Se estima que en Ecuador, aproximadamente el $40 \%$ de la producción de oro deriva de procesos de amalgamación y el $60 \%$ de cianuración; por otro lado, de aquellos mineros que todavía utilizan amalgamación, un $50 \%$ emplean retorta y un 50\% eliminan el mercurio mediante soplete a cielo abierto, muchas veces en sus domicilios (Resabala, 2008). Sin embargo, los sistemas empleados son poco eficientes y dejan escapar vapores de mercurio al ambiente, llegando a constituir un riesgo en la salud de los mineros y trabajadores, de no tomarse las precauciones necesarias, ya que estos vapores pueden ser absorbidos por diferentes vías, entre ellas, la inhalación.

\subsection{Impactos de la actividad}

La emisión de mercurio por la actividad minera según el inventario nacional de emisiones de mercurio del Ministerio de Ambiente del Ecuador (2008), estima que hacia 2005 se pudieron liberar al medio ambiente más de $3200 \mathrm{~kg}$ de mercurio elemental (Resabala, 2008), lo cual representa un alto grado de contaminación de las área mineras, y exposición de los trabajadores y personas que habitan las zonas en cuestión. En términos de normativa, se puede indicar que para el vapor de mercurio $(\mathrm{Hg})$ en aire ambiente no existe un límite máximo establecido por las leyes ecuatorianas, por ende solo se dispone de referencias internacionales para fines investigativos; así pues los límites máximos admisibles para el mercurio en forma de vapor son: TLV-TWA (Threshold Limit Value) $=0,025 \mathrm{mg} \mathrm{m}^{-3}$, establecido por la American Conference of Governmental Industrial Hygienists ACGIH (OSHA, 2004); así como REL (Recommended Exposure Limit) $=0,050 \mathrm{mg} \mathrm{m}^{-3}$, establecido por National Institute for Occupational Safety and Health (NIOSH) (International Chemical Safety Cards, 2004); se dispone además de un valor referencial de la Organización Mundial de la Salud (OMS) que recomienda un máximo de $1 \mu \mathrm{g} \mathrm{m}^{-3}$ como promedio anual (WHO, 2007). El gran impacto de la exposición prolongada a los vapores de mercurio sobre la salud de los mineros está bien descrito en la literatura médica, conocida como "hidrargirismo" que genera efectos crónicos sobre el sistema nervioso central (SNC) y el aparato urinario principalmente; hay pocos datos disponibles acerca del efecto sobre otros órganos, como el sistema circulatorio, o el riesgo de cáncer por la exposición al mercurio. Estos efectos son potencialmente dañinos sobre mujeres embarazadas, el feto en gestación y en los niños. En la exposición a mercurio elemental, o sea aquella a la que están expuestos los mineros auríferos, se puede generar déficit en el desarrollo neurológico y de comportamiento, esto incluiría un daño en la memoria, atención y respuesta, capacidad auditiva y psicomotoras, pérdida reversible de la capacidad para distinguir colores, además de inflamaciones severas de la piel, entre otros efectos (Olivero y col., 2002). Algunas investigaciones en otros países sobre comunidades mineras de pequeña escala, han demostrado que la exposición a los vapores de mercurio provenientes de procesos artesanales de quema de amalgama pueden ser altamente nocivos para el ser humano (Bose-O'Reilly y col., 2008; Paruchuri y col., 2010). En Ecuador han existido iniciativas de mejora de las condiciones de trabajo de la minería, algunos basados en asistencia técnica internacional tales como los proyectos llevados a cabo por CENDA/COSUDE y PRODEMINCA, en las zonas de Portovelo y Zaruma, reportando interesantes resultados, de igual manera iniciativas estatales de CODIGEM, INEMIN y DINAGE, así como otras de índole privada, que también han fomentado buenas prácticas productivas y capacitación en el sector (Sandoval, 2001). 


\subsection{Objetivos del estudio}

El propósito de este estudio es brindar una visión y acercamiento a la realidad del nivel de riesgo al que están expuestos los trabajadores de algunas áreas mineras del Cantón Ponce Enríquez, debido al uso de mercurio en la extracción de oro, y conocer si las concentraciones de vapor de mercurio en los procesos de quema de amalgama muestran niveles riesgosos para la salud, así como su distribución en la zona de trabajo durante el proceso; para ello se empleó un análisis instrumental in situ para detección de concentraciones, utilizando técnica de tubos Colorimétricos GASTEC de Japón (GASTEC Corp., 2008) en los ambientes laborales muestreados y definiendo distancias de muestreo desde el punto focal de emisión de vapor de mercurio; el estudio se basó en cuatro campamentos mineros de la zona minera de Bella Rica (Ponce Enríquez), los que fueron escogidos por un muestreo sesgado, debido a la limitada apertura de varias de las sociedades mineras a ser partícipes de la investigación.

\section{MATERIALES Y MÉTODOS}

Se empleó un diseño experimental aleatorio, descriptivo y analítico (Hernández y col., 2007). Para la recolección de datos experimentales se seleccionaron 4 campamentos mineros (por muestreo sesgado) localizados en la comunidad de Bella Rica de Ponce Enríquez, en la Provincia del Azuay, que se sitúan entre los 835 y $1057 \mathrm{msnm}$ a una distancia de 17,5 km desde Ponce Enríquez (Latitud $3^{\circ} 04^{\prime} 12^{\prime \prime}$ S - Longitud: $79^{\circ} 41^{\prime} 56^{\prime \prime O}$ ). El muestreo durante los procesos de quema de amalgama se realizó bajo las dos condiciones de trabajo observadas: quema directa de la amalgama y ataque previo con ácido nítrico $\left(\mathrm{HNO}_{3}\right)$ concentrado + quema directa, estos métodos se describen a continuación:

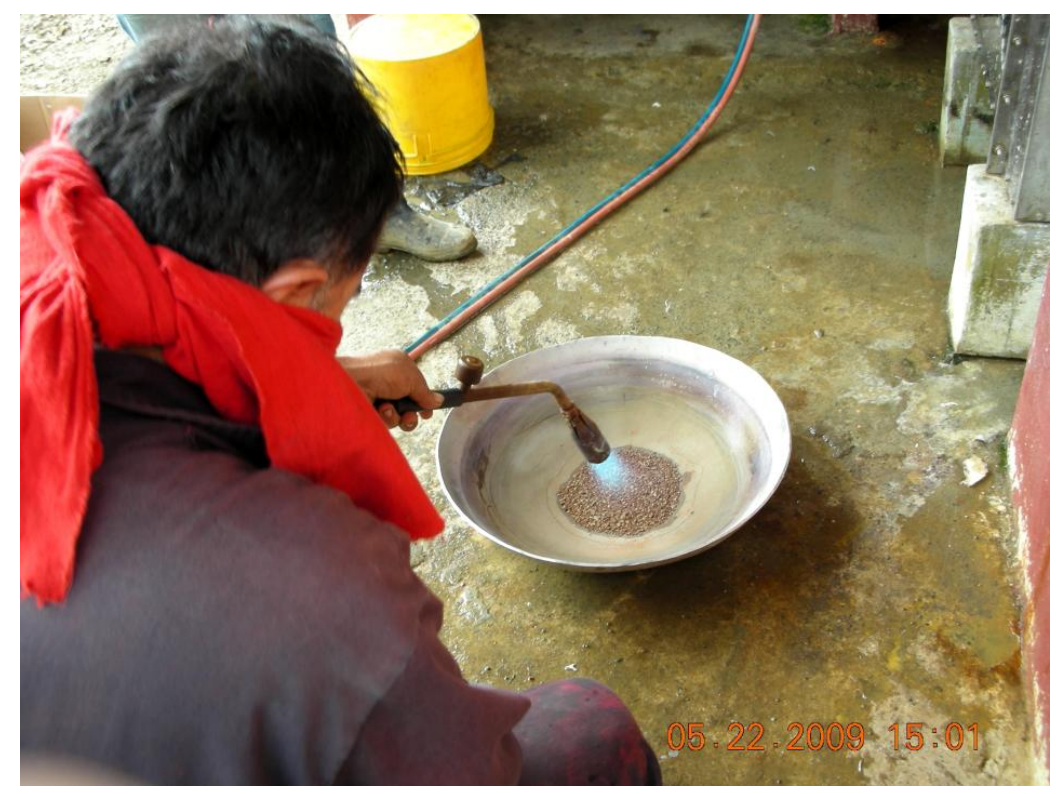

Figura 1. Fotografía del proceso de quema de amalgama en uno de los campamentos.

La quema directa consiste en un solo tratamiento de la amalgama de mercurio-oro, la cual se deposita en una retorta o plato metálico y se aplica una llama directa de soplete de gas propano (GLP) hasta evaporar totalmente el mercurio, quedando una esponja de oro la cual se refinará posteriormente. La duración y eficiencia del proceso varía de acuerdo a la experiencia del trabajador. El ataque previo con ácido nítrico $\left(\mathrm{HNO}_{3}\right)$ concentrado + quema directa con soplete sobre la amalgama, es un doble tratamiento de la amalgama donde a la misma se la somete de modo preliminar a un ataque con ácido nítrico de alta concentración (industrial), el cual aporta con la 
disolución de óxidos metálicos e impurezas que se encuentren en la amalgama, se desecha el ácido después de un breve periodo de tiempo y se procede a quemar la amalgama con el método anteriormente mencionado. En la Figura 1 se puede observar el proceso de quema de amalgama en uno de los campamentos investigados.

Para la determinación de los niveles de vapor de mercurio en las zonas de trabajo, se consideró un modelo de muestreo a distancias concéntricas alrededor del punto de quema (a 0,00;0,20;0,50; 1,00 y $2,00 \mathrm{~m}$ de distancia y en una distribución de $0,90,180,270$ y $360^{\circ}$ alrededor del punto en estudio), se procedió a tomar una muestra de aire por duplicado en cada distancia y ángulo, para un total de cinco muestras con duplicado para cada proceso, además de una muestra denominada blanco, en un punto alejado del área (a una distancia mayor a $50 \mathrm{~m}$ ). La recolección de muestras se realizó de tal manera, que se abarque toda el área a la cual están expuestos directamente los trabajadores. Se registró también las condiciones ambientales con la finalidad de realizar las correcciones necesarias. Todos los ambientes donde se efectuaron los muestreos de los procesos estaban al interior de las edificaciones de cada campamento, su estructura era parcialmente cubierta y con condiciones similares de ventilación y temperatura, por lo que en estas condiciones se asume que la distribución del vapor de mercurio se dio de modo horizontal, en todas las direcciones, sin perturbaciones, ni turbulencia significativa en el aire ambiente de cada sitio. Los campamentos fueron codificados como $\mathrm{CP} 1, \mathrm{CP} 2, \mathrm{CP} 3$ y CP4 tienen características peculiares y condiciones de trabajo específicas que se detallan en la Tabla 1.

Para la determinación de la concentración de vapor de mercurio en el aire ambiente, se utilizó una bomba de precisión volumétrica GASTEC GV100 y tubos detectores GASTEC \#40 de cristal, con lectura directa de concentración en mg de vapor de mercurio por metro cúbico de aire ambiente (mg Vap.Hg m${ }^{-3}$ aire). La detección de vapor de mercurio en el aire se la realiza por toma de muestra en volumen estándar de $100 \mathrm{ml}$ con la bomba de precisión volumétrica y tubos detectores de cristal GASTEC \#40 que contienen yoduro de cobre (CuI) en lámina de absorción prefabricada. El principio de reacción es el siguiente:

$$
\mathrm{Hg}+4 \mathrm{CuI} \rightarrow \mathrm{Cu}_{2}\left(\mathrm{HgI}_{4}\right)+2 \mathrm{Cu}
$$

$\mathrm{El}$ complejo $\mathrm{Cu}-\mathrm{Hg}$ formado posee un color amarillo pálido, de directa diferenciación visual en una escala de concentración que muestran los tubos GASTEC \#40. El límite de detección de estos

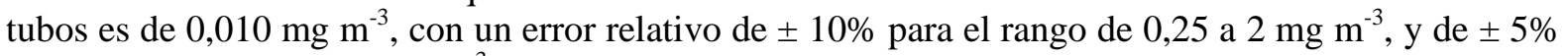
para el rango de 2 a $6 \mathrm{mg} \mathrm{m}^{-3}$ (GASTEC Corp., 2008). La lectura de los tubos se ve afectada por fluctuaciones significativas de la presión atmosférica, los tubos están calibrados a una presión atmosférica normal de $1013 \mathrm{hPa}(760 \mathrm{~mm} \mathrm{Hg})$. Para corregir la presión atmosférica se empleó la siguiente fórmula:

$$
\text { Valor corregido }\left(\frac{\mathrm{mg}}{\mathrm{m}^{3}}\right)=\frac{\text { Valor muestreado }\left(\frac{\mathrm{mg}}{\mathrm{m}^{3}}\right) \times 1013(\mathrm{hPa})}{\text { Presión atmosférica del punto de muestreo }(\mathrm{hPa})}
$$

No se requirió corrección de temperatura, ni humedad. De forma análoga, se recurrió a la observación, la misma que permitió recolectar datos referentes a la manipulación del mercurio durante el proceso, y con la finalidad de evidenciar o no, el uso adecuado del equipo de protección personal. Se registró los valores de concentración de mercurio en el aire ambiente, las distancias de monitoreo y los tiempos de exposición al vapor del mercurio. Es importante mencionar que debido a la técnica de análisis empleada (que requiere de un cambio de tubo colorimétrico para cada muestra) el registro de tiempo de muestreo tuvo una variación con cada muestra tomada; sin embargo, la misma es poco significativa en la continuidad del monitoreo. Se consideró emplear esta técnica por su economía, facilidad de uso y portabilidad en lugares de difícil acceso. El tiempo de duración de los procesos de ataque ácido mas quema de amalgama fluctuó entre 16 y $38 \mathrm{~min}$, mientras que en los de quema de amalgama directa entre 17 a $23 \mathrm{~min}$. Los datos obtenidos se analizaron por métodos de regresión para hallar tendencias y comportamiento entre las variables, con análisis del coeficiente de determinación (Stacey y col., 2007). Se empleó la observación detallada de los procesos y el registro fotográfico para interpretar los resultados. 
MASKANA, Vol. 2, No. 2, 2011

Tabla 1. Características generales de los campamentos mineros y condiciones de proceso de vaporización de amalgama Au-Hg.

\begin{tabular}{|c|c|c|c|c|c|c|}
\hline $\begin{array}{l}\text { Código del } \\
\text { campamento }\end{array}$ & $\begin{array}{c}\text { Área aprox. de } \\
\text { zona de quema de } \\
\text { amalgama }\left(\mathrm{m}^{2}\right)\end{array}$ & $\begin{array}{l}\text { Tratamiento de la } \\
\text { amalgama }\end{array}$ & $\begin{array}{c}\text { Características de } \\
\text { cubierta de la zona } \\
\text { de trabajo }\end{array}$ & $\begin{array}{l}\text { Condiciones de viento y } \\
\text { movimiento de aire }\end{array}$ & $\begin{array}{l}\text { Humedad }(\%) \mathrm{y} \\
\text { temperatura }\left({ }^{\circ} \mathrm{C}\right)\end{array}$ & Ubicación \\
\hline CP1 & 4 & $\begin{array}{l}\text { - Quema directa de } \\
\text { la amalgama con } \\
\text { soplete }\end{array}$ & $\begin{array}{l}\text { Parcialmente } \\
\text { cubierta }\end{array}$ & $\begin{array}{l}\text { Viento nulo, no se percibe } \\
\text { turbulencias de aire }\end{array}$ & $\begin{array}{l}80 \sim 90 \% \\
21 \sim 25^{\circ} \mathrm{C}\end{array}$ & $\begin{array}{l}3^{\circ} 04^{\prime} 44^{\prime \prime} \mathrm{S}, \\
79^{\circ} 42^{\prime} 04^{\prime \prime} \mathrm{O}\end{array}$ \\
\hline $\mathrm{CP} 2$ & 6 & $\begin{array}{l}\text { Quema directa de la } \\
\text { amalgama con soplete } \\
\text { - Ataque con ácido } \\
\text { nítrico + quema de la } \\
\text { amalgama con soplete }\end{array}$ & $\begin{array}{l}\text { Parcialmente } \\
\text { cubierta }\end{array}$ & $\begin{array}{l}\text { Viento nulo, no se percibe } \\
\text { turbulencias de aire }\end{array}$ & $\begin{array}{l}80 \sim 90 \% \\
21 \sim 25^{\circ} \mathrm{C}\end{array}$ & $\begin{array}{l}3^{\circ} 04^{\prime} 45^{\prime \prime} \mathrm{S}, \\
79^{\circ} 42^{\prime} 04^{\prime \prime} \mathrm{O}\end{array}$ \\
\hline CP3 & 6 & $\begin{array}{l}\text { Quema directa de la } \\
\text { amalgama con soplete } \\
\text { - Ataque con ácido } \\
\text { nítrico + quema de la } \\
\text { amalgama con soplete }\end{array}$ & $\begin{array}{l}\text { Parcialmente } \\
\text { cubierta }\end{array}$ & $\begin{array}{l}\text { Viento nulo, no se percibe } \\
\text { turbulencias de aire }\end{array}$ & $\begin{array}{l}80 \sim 90 \% \\
18 \sim 23^{\circ} \mathrm{C}\end{array}$ & $\begin{array}{l}3^{\circ} 04^{\prime} 14^{\prime \prime} \mathrm{S} \\
79^{\circ} 41^{\prime} 58^{\prime \prime} \mathrm{O}\end{array}$ \\
\hline $\mathrm{CP} 4$ & 7 & $\begin{array}{l}\text { Quema directa de la } \\
\text { amalgama con soplete } \\
\text { - Ataque con ácido } \\
\text { nítrico + quema de la } \\
\text { amalgama con soplete }\end{array}$ & $\begin{array}{l}\text { Parcialmente } \\
\text { cubierta }\end{array}$ & $\begin{array}{l}\text { Viento nulo, no se percibe } \\
\text { turbulencias de aire }\end{array}$ & $\begin{array}{l}80 \sim 90 \% \\
18 \sim 23^{\circ} \mathrm{C}\end{array}$ & $\begin{array}{l}3^{\circ} 04^{\prime} 14^{\prime \prime} \mathrm{S} \\
79^{\circ} 41^{\prime} 57^{\prime \prime} \mathrm{O}\end{array}$ \\
\hline
\end{tabular}




\section{RESULTADOS Y DISCUSIÓN}

Se procedió a graficar los valores de concentración de mercurio corregidos de las muestras tomadas en los campamentos CP1, CP2, CP3 y CP4, con su respectiva regresión para hallar comportamientos de la concentración versus la distancia de la fuente de emisión (punto de tratamiento térmico de la amalgama) (ver Figura 2).
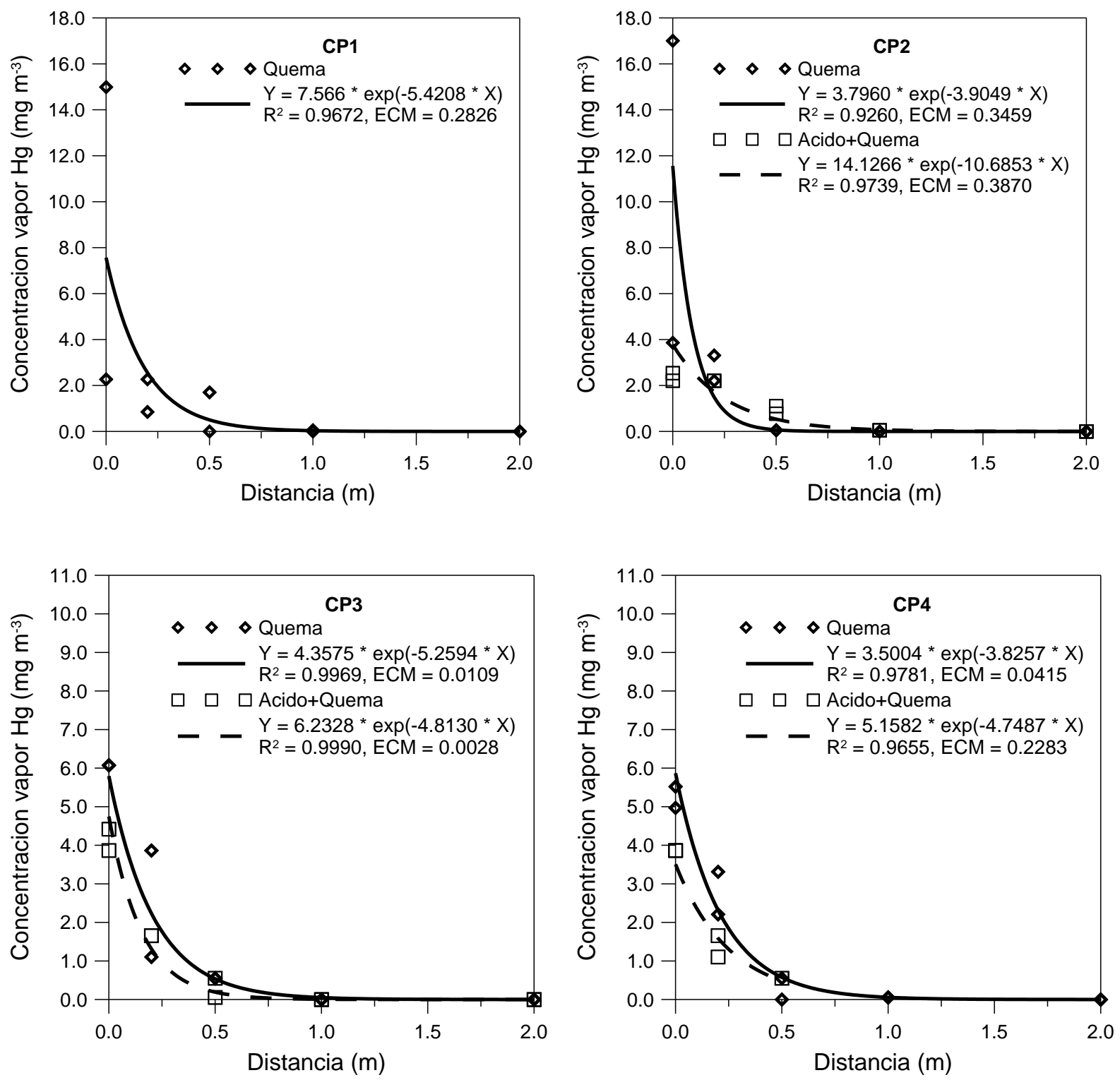

Figura 2. Evolución de la concentración de vapor de $\mathrm{Hg}$ en función de la distancia de la fuente de generación, para los cuatro campamentos mineros $\left(\mathrm{CP} 1^{1}, \mathrm{CP} 2, \mathrm{CP} 3\right.$ y $\left.\mathrm{CP} 4\right)$ investigados.

Se puede apreciar mediante el análisis de modelos de regresión lo siguiente: para el Campamento CP1donde se analiza únicamente el proceso de quema de amalgama, se observa una tendencia exponencial de concentración, la cual reduce sus valores en función de la distancia, el coeficiente de

\footnotetext{
${ }^{1}$ En el primer campamento CP1, no existe tratamiento de ataque ácido + quema de amalgama.
} 
determinación para este comportamiento es elevado $\left(\mathrm{R}^{2}=0,9672\right)$ lo cual lo hace muy significativo. El error cuadrático medio de menor grado se ajusta a una curva tipo exponencial. Se puede apreciar que la concentración tiende a cero cuando la distancia es superior a $1 \mathrm{~m}$, por lo que a partir de esa distancia no se supera el límite NIOSH para vapores de mercurio y por ende puede considerarse una distancia segura de trabajo. En el Campamento CP2 se tratan los dos procesos, con quema directa y ataque ácido mas quema, en ambos casos, el comportamiento se apega a una tendencia exponencial de concentración, la cual tiene un decrecimiento frente a la distancia, con coeficientes de determinación significativos $\left(\mathrm{R}^{2}=0,9260\right.$ y 0,9739$)$. Ambas tendencias son similares y definen que la distancia mínima, donde la concentración de vapor de mercurio que tiende a cero, es de $1 \mathrm{~m}$ en adelante. Para el Campamento CP3 se hallan condiciones muy similares a los anteriores campamentos, pero con coeficientes de determinación muy elevados $\left(\mathrm{R}^{2}=0,9969\right.$ y 0,9990$)$ y error cuadrático medio muy bajo en comparación con los otros casos, lo cual demuestra igualmente que la tendencia es exponencial inversa y que las distancias superiores a $1 \mathrm{~m}$ son seguras pues su concentración de vapor de mercurio tiende a cero. Igual caso se tiene con el Campamento CP4 el cual también tiene coeficientes de determinación elevados $\left(\mathrm{R}^{2}=0,9781\right.$ y 0,9655$)$ y muestra que distancias de un metro en adelante tienen concentraciones mínimas o nulas.

Se confirma por lo tanto que los vapores de mercurio, ya sea bajo el proceso de quema directa de amalgama o ataque de ácido nítrico mas quema de amalgama, se dispersan significativamente en la atmósfera de trabajo en distancias menores a un metro, siendo una distancia mayor a esta representativamente segura para el trabajo. Este hallazgo es considerado única y exclusivamente bajo las condiciones que se han presentado en los campamentos investigados (ver Tabla 1) y no se puede considerar como una condición que excluye la responsabilidad del uso del equipo de protección respiratoria por parte de los trabajadores que realizan este tipo de actividad. En condiciones atmosféricas diferentes, particularmente de presencia de viento influyente y elevado movimiento o turbulencias del aire en la zona de trabajo pudieran manifestarse diferentes comportamientos del vapor de mercurio, facilitando su dispersión a distancias superiores a las halladas en el presente estudio. Si se compara el valor límite NIOSH versus las concentraciones encontradas, se observa que estas superan en decenas y hasta cientos de veces este límite, particularmente en aquellas distancias entre 0,00 y $0,50 \mathrm{~m}$, lo cual es muy riesgoso para los trabajadores que se exponen a estas emanaciones del proceso. Se ha observado que los trabajadores mantienen distancias muy próximas a las mencionadas, durante la actividades de quema en todos los sitios investigados, tal como puede apreciarse en la fotografía del proceso de quema de amalgama en uno de los campamentos (Figura 1); y adicionalmente, emplean solo una tela o franela para prevenir la inhalación de los vapores, no emplean guantes, ni vestimenta impermeable, lo cual permite la condensación del vapor de mercurio sobre las superficies absorbentes como el algodón y directamente sobre la piel y cabello. Los resultados de concentración en las distancias menores a un metro, tanto para quema directa y quema con ataque previo de ácido nítrico, se muestran notablemente elevados y son potencialmente riesgosos para la salud de los trabajadores por superar los límites referenciales. Otros autores han reportado concentraciones de vapor de mercurio en aire ambiente laboral de hasta $50 \mu \mathrm{g} \mathrm{m}^{-3}\left(0,050 \mathrm{mg} \mathrm{m}^{-3}\right)$ en Manica, Mozambique (Swain y col., 2007; UNIDO, 2005) y de hasta $150.000 \mathrm{ng} \mathrm{m}^{-3}\left(0,150 \mathrm{mg} \mathrm{m}^{-3}\right)$, en Portovelo-Zaruma, Ecuador (Velásquez-López y col., 2010), determinados bajo metodologías de análisis distintas (espectrometría de absorción atómica portátil) en campamentos donde se realizan actividades de quema de amalgama bajo similares condiciones. En ambas referencias los valores superan los límites referenciales establecidos por la NIOSH y WHO. La dispersión horizontal del vapor de mercurio en áreas del ambiente laboral facilita la precipitación de mercurio en forma de condensado sobre el suelo, infraestructura y herramientas de trabajo, así como también en cuerpos de agua y vegetación cercana, lo cual es un riesgo muy serio de exposición para las personas que transitan por la zona contaminada, según se ha podido observar. Otras investigaciones han demostrado y estudiado más a fondo este efecto negativo de precipitación de condesados de mercurio sobre superficies circundantes a zonas de quema de amalgama (Babut y col., 2003), y el mismo es muy alarmante. En base a la observación de los procesos, se puede confirmar que la práctica de quema de amalgama en ambientes cerrados o abiertos, muestra un elevado riesgo de afectación a la salud por la dispersión de los vapores de mercurio; por ello, siguiendo ciertas pautas internacionales de manejo de mercurio a pequeña escala, se debe considerar que ninguna persona debería quemar o calentar 
amalgama de mercurio para recuperar oro sin la utilización de un medio adecuado y tecnológicamente eficiente para contención, condensación y recuperación de los vapores mercuriales; el trabajador debería mantener distancias de seguridad adecuadas (superiores a un metro de distancia, en ambientes de interiores con ventilación), emplear un adecuado equipamiento de seguridad respiratoria y vestimenta, y realizar la actividad en zonas alejadas de viviendas; así como también, restringir la presencia de niños o mujeres embarazadas durante el proceso (Spiegel y Veiga, 2010). Se ha observado que en ninguno de los campamentos se cumplen estas condiciones (exceptuando la prohibición de presencia de niños o mujeres embarazadas), lo cual es una situación muy preocupante.

\section{CONCLUSIONES}

Al cuantificar analíticamente las concentraciones de vapor de mercurio en los campamentos mineros investigados, se observan niveles en la atmósfera laboral que superan en gran medida los límites referenciales sugeridos por ACGIH y NIOSH; los valores reportados pueden exceder en decenas o cientos de veces estos límites mientras mayor proximidad se tenga con la fuente de emisión (punto de quema de la amalgama). El análisis de emisiones de vapor $\mathrm{Hg}$, muestra que en la zona propia de trabajo, las concentraciones son considerablemente elevadas entre 0,00 a $0,50 \mathrm{~m}$ desde el punto focal de quema, pero sus valores disminuyen notablemente a partir de $1,00 \mathrm{~m}$ de distancia en adelante, considerándose ésta una distancia libre de vapores detectables en la mayoría de los casos investigados; sin embargo, esto no se lo puede considerar como una recomendación, ni como una regla para favorecer la continuidad de aplicación de esta técnica artesanal en la minería de pequeña escala, sobre todo considerando que los trabajadores no están habituados al uso continuo de equipo de seguridad respiratorio, ni ropa y guantes protectores adecuados, además los resultados del estudio son válidos cuando estos procesos de quema de amalgama se llevan a cabo en áreas interiores de los campamentos con estructuras parcialmente cubiertas, que favorecen una ventilación sin perturbaciones significativas en el movimiento del aire. En base a los hallazgos de la investigación, la quema de amalgama de mercurio que prescinda de medios de contención, tales como retortas cerradas con sistema de condensación y recuperación del mismo, debe considerarse una práctica de alto riesgo para la salud de los trabajadores y de grave afectación del medioambiente.

Cabe destacar también, que al momento no existe una normativa ecuatoriana específica para niveles de vapor de mercurio en aire ambiente, lo cual es una falencia desde el punto de vista de control ambiental y de seguridad y salud en el trabajo; solo se dispone de niveles referenciales sugeridos por organismos internacionales para este tipo de investigaciones. Al respecto de las posibilidades de reemplazo de estas prácticas artesanales de extracción, se puede indicar que hay un considerable grado de desconocimiento de tecnologías alternativas por parte de los trabajadores, así como un desinterés en el cambio que podría mejorar las condiciones laborales y ambientales. La Organización de Naciones Unidas en su programa "Removal of Barriers to the Introduction of Cleaner Artisanal Gold Mining and Extraction Technologies" o conocido también como Global Mercury Project (GMP) ha reportado que para la aplicación de tecnologías 100\% libres de mercurio (tales como, cianuración) se requiere un elevado capital económico, entrenamiento y organización, que aquellas sociedades mineras más pobres no pueden costear (UNIDO, 2007), por ende es muy importante considerar que el apoyo gubernamental para nuevas iniciativas de mejora tecnológica deben alcanzar prioritariamente a las sociedades mineras de menor escala y las más pobres, por ser las más propensas a seguir empleando estas técnicas rudimentarias de extracción de oro. El planteamiento de diseño y uso de sistemas de recuperación del mercurio de fabricación económica, así como la concientización y difusión de los factores de riesgo en estos procesos empíricos, son alternativas viables de ejecución en mediano plazo para una mitigación del problema. La presente investigación no ha abordado el aspecto de análisis clínico de detección de mercurio en sangre, orina y cabello (biomonitores), lo cual ampliaría de mejor manera el alcance del estudio y sus resultados como lo han demostrado otras investigaciones que se enfocan en estos aspectos (Bose-O'Reilly y col., 2008). Queda abierta la posibilidad de investigar más a fondo esta problemática, ahondando en el campo médico, con la investigación de niveles de intoxicación por mercurio en los mineros y sus familias, así 
como el estudio de otros elementos contaminantes del aire ambiente de estas zonas; tales como: metales pesados nocivos (ej., arsénico, cobre, plomo, etc.); y emisiones gaseosas tóxicas provenientes de las fuentes fijas de combustión empleadas en el proceso (compresores y bombas accionados por motores de gasolina o diesel), las que generalmente se encuentran muy cerca de las zonas de trabajo, descanso, alimentación y vivienda de los trabajadores; según lo que se ha podido observar en el estudio.

\section{AGRADECIMIENTO}

El presente estudio se ha desarrollado en el contexto del Proyecto: "Estudio de la Generación de Vapores de Mercurio en la Extracción de Oro, y sus Efectos en los Trabajadores de una Área Minera en el Cantón Ponce Enríquez, Azuay", financiado por la Dirección de Investigación de la Universidad de Cuenca (DIUC). Los autores desean expresar su agradecimiento a la Universidad de Cuenca, Dirección de Investigación de la Universidad de Cuenca (DIUC) y Centro de Estudios Ambientales (CEA) en la persona de la Dra. Nancy García por su asesoría, apoyo y respaldo institucional prestado en esta investigación, la cual pretende generar conciencia sobre la gravedad de las condiciones de la contaminación producida por los métodos de producción minera artesanal que aún existen en nuestro país, y establecer pautas para un cambio sostenible. De igual manera deseamos expresar un agradecimiento al Dr. Jan Feyen por su gentil apoyo en la revisión del presente artículo.

\section{BIBLIOGRAFÍA}

Babut, M., R. Sekyi, A. Rambaud, M. Potin-Gautier S. Tellier W. Bannerman, C. Beinhoff, 2003. Improving the environmental management of small-scale gold mining in Ghana: a case study of Dumasi. J. Cleaner Prod., 11, 215.

Bose-O'Reilly, S., B. Lettmeier, R. Matteucci Gothe, C. Beinhoff, U. Siebert, G. Drasch, 2008. Mercury as a serious health hazard for children in gold mining areas. Environ. Res., 107, 89-97.

Cisneros, P., 2008. El diálogo minero en el Ecuador - ¿Señales de una nueva relación entre comunidades, empresas extractivas y estado?. Descargado de http://www.flacso.org.ec/ docs/WP_012_Cisneros_02.pdf el 15 de septiembre de 2011.

GASTEC Corp., 2008. Environmental analysis technology. GASTEC Handbook (8th Ed.), McGraw Hill, NY, USA, 350págs.

Hernández, R., C. Fernández, P. Baptista, 2007. Metodología de la investigación (4 Ed.). McGraw Hill, México D.F., México, 45-805.

INEC, 2001. Base de Datos REDATAM de la provincia del Azuay 1990-2001. Universidad de Cuenca, Cuenca, Ecuador.

International Chemical Safety Cards, 2004. ICSC 0056: Mercury. Descargado de http://www.cdc.gov/niosh/ipcsneng/neng0056.html el 10 de junio de 2010.

Ministerio de Ambiente del Ecuador, 2008. Inventario nacional de emisiones de mercurio y productos que contienen mercurio. Ministerio de Ambiente del Ecuador, Quito, Ecuador, 221 págs.

MEM (Ministerio de Energía y Minas del Ecuador), 2007. El ABC de la minería en el Ecuador, Quito. Descargado de http://infomineria.org/fileadmin/download/ABCdelaMineria.pdf el 14 de septiembre de 2011.

OSHA (Occupational Safety \& Health Administration), 2004. Safety and health topics: Mercury (Vapor). Descargado de http://www.osha.gov/dts/chemicalsampling/data/CH_250510.html el 10 de junio de 2010.

Olivero, J., B. Johnson, 2002. El lado gris de la minería del oro: La contaminación con mercurio en el norte de Colombia. En: J.Matson (Ed.), Universidad de Cartagena, Cartagena D.C., Colombia. 
Paruchuri, Y., A. Siuniak, N. Johnson, E. Levin, K. Mitchell, J.M. Goodrich, E.P. Renne, N. Basu, 2010. Occupational and environmental mercury exposure among small-scale gold miners in the Talensi-Nabdam District of Ghana's Upper East region. Sci. Total Environ., 408, 6079-6085.

PNUMA, 2005. Instrumental para la identificación y cuantificación de liberaciones de mercurio, Borrador Preliminar, Ginebra. Descargado de http://www.zeromercury.org/UNEP_developments/ studyglobaloptionsES.pdf el 15 de septiembre de 2011.

Resabala, C., 2008. Informe final del inventario nacional de emisiones de mercurio y productos que contienen mercurio. Ministerio del Ambiente del Ecuador. Quito, Ecuador, 60 págs.

Sandoval, F., 2001. La Pequeña Minería en el Ecuador. En: IIED and WBCSD (Ed.), Mining, Minerals and Sustainable Development (MMSD), 75, 30 págs.

Spiegel, S J., M.M. Veiga, 2010. International guidelines on mercury management in small-scale gold mining. J. Cleaner Prod., 18, 375-385.

Stacey J., L.T. Shaefer, 2007. Probability and statistics: Applications for environmental science. CRC Press Taylor \& Francis Group, USA, 367 págs.

Swain, E.B., P.M. Jakus, G. Rice, F. Lupi, P.A. Maxson, J.M. Pacyna, A. Penn, S.J. Spiegel, M.M Veiga, 2007. Socioeconomic consequences of mercury use and pollution. J. Human Environ., 36, 45-61.

UNIDO, 2005. United Nations Industrial Development Organization: Pilot project for the reduction of mercury contamination resulting from artisanal gold mining fields in the Manica District of Mozambique. Report to UNIDO and Blacksmith Institute, 43 págs.

UNIDO, 2007. United Nations Industrial Development Organization: Global impacts of mercury supply and demand in small-scale gold mining. En: Report to the United Nations Environment Program Governing Council Meeting, Nairobi, Kenya, 9 págs. Descargado de http://www.unep.org/hazardoussubstances/Mercury/tabid/434/language/en-US/Default.aspx el 15 de septiembre de 2011.

Velásquez-López, P.C., M.M. Veiga, K. Hall, 2010. Mercury balance in amalgamation in artisanal and small-scale gold mining: Identifying strategies for reducing environmental pollution in Portovelo-Zaruma, Ecuador. J. Cleaner Prod., 18, 226-232.

WHO, 2007. World Health Organization. Exposure to mercury: A major public health concern. Documento PDF. Geneva, Switzerland, 4 págs. Descargado de http://www.who.int/phe/ news/Mercury-flyer.pdf el 15 de septiembre de 2011. 